\title{
Prevalence and type distribution of human papillomavirus in 5,000 British Columbia women-implications for vaccination
}

\author{
Richard A. Moore · Gina Ogilvie · Daniel Fornika · Veronika Moravan · \\ Marc Brisson - Mahsa Amirabbasi-Beik - Anita Kollar - Thomas Burgess • \\ Ray Hsu · Laura Towers · Jane Lo · Jasenka Matisic · Angela Brooks-Wilson
}

Received: 9 June 2008/ Accepted: 6 May 2009/Published online: 29 May 2009

(C) The Author(s) 2009. This article is published with open access at Springerlink.com

\begin{abstract}
Background Human papilloma virus (HPV) prevalence studies performed in different regions and population groups across Canada would inform public health decisions regarding implementation of anti-HPV vaccines.

Methods A total of 8,700 liquid-based specimens from 8,660 women aged 13-86 from throughout British Columbia were collected. DNA was isolated from 4,980 of these samples and assessed for HPV prevalence and type
\end{abstract}

Electronic supplementary material The online version of this article (doi:10.1007/s10552-009-9365-4) contains supplementary material, which is available to authorized users.

R. A. Moore - D. Fornika · M. Amirabbasi-Beik · A. Kollar ·

T. Burgess · R. Hsu · A. Brooks-Wilson $(\square)$

Canada's Michael Smith Genome Sciences Centre, British

Columbia Cancer Agency, 675 West 10th Ave., Vancouver,

BC V5Z 1L3, Canada

e-mail: abrooks-wilson@bcgsc.ca; awilson@bcgsc.ca

V. Moravan $\cdot$ L. Towers $\cdot$ J. Lo $\cdot$ J. Matisic

Cervical Cancer Screening Program, British Columbia

Cancer Agency, 686 West Broadway, Vancouver,

BC, Canada

G. Ogilvie

British Columbia Centre for Disease Control, 655 West

12th Ave., Vancouver, BC, Canada

G. Ogilvie

The Department of Family Practice, University of British

Columbia, Vancouver, BC, Canada

A. Brooks-Wilson

Department of Biomedical Physiology and Kinesiology,

Simon Fraser University, Vancouver, BC, Canada

M. Brisson

Laval University, Quebec City, QC, Canada distribution. HPV was detected by PCR analysis using tagged GP5+/6+ consensus primers to amplify the L1 region of HPV; typing was done by bi-directional sequencing of PCR products.

Results Overall HPV prevalence was $16.8 \%$ (age adjusted $15.5 \%$ ). Prevalence of high-risk HPV was 13.9, and $10.7 \%$ of samples contained HPV16. HPV prevalence was highest in the youngest group of women $(<20$ years). Onethird of HPV positive samples contained more than one HPV type. Percentages of low-grade (LGIL) and highgrade intraepithelial lesions (HGIL) containing high-risk HPV are 52.3 and $79.4 \%$, respectively.

Conclusions Overall HPV prevalence in this study is within the range of estimates from other studies. The prevalence of HPV16 is higher than what is found in other Canadian and international studies. HPV16 and HPV18 compose a majority of the high-risk virus in this study. Use of current HPV vaccines could considerably reduce HPVrelated conditions including cervical cancer and procedures such as colposcopy.

Keywords HPV · Typing · Prevalence $\cdot$ Canada

\section{Introduction}

Human papillomavirus (HPV) is now understood to be necessary but insufficient for the development of cervical cancer [1]. There are more than 100 known types of HPV, of which over 40 infect the female genital tract. Of these, at least 15 are denoted as 'high risk' (HR) [2] for cervical cancer.

The recent development of vaccines against two (HPV types 16 and 18) [3,4] or four (HPV types 16, 18, 6, and 11) [5-7] HPV types has highlighted the need for timely 
population-based HPV prevalence data for Canada. Such data can be used to estimate the expected effectiveness of these vaccines in reducing conditions and procedures arising from those HPV types. It can also establish a baseline from which to monitor potential changes in HPV prevalence and type distribution after uptake of a vaccine. Our objective was to establish baseline HPV prevalence and HPV type distribution in women who participated in the population-based cervical cytology screening program in British Columbia in 2004, to enable optimal public health decision-making regarding prevention of cervical cancer and related conditions.

\section{Methods}

\section{Study population}

The centralized cervical cancer screening program (CCSP) of $\mathrm{BC}$ has been operational since 1960. It processes every Pap smear done in $\mathrm{BC}$ at a single facility; all cytology results are stored in a single database. More than half a million women participate in the CCSP each year and over $70 \%$ of eligible women in $\mathrm{BC}$ are screened, on average, every 30 months.

\section{Specimen collection and cytological interpretation}

A flowchart summarizing sample collection and experiments is shown in Supplemental Online Figure A. The 8,700 samples used in this study were derived from a feasibility study of liquid-based smears collected by 99 high-volume smear-takers from different parts of BC within the CCSP between March and July 2004 [8]. The sample included women aged 13-86; median age was 38 . About $98.2 \%$ of the smears in this study are from the cervix or endocervix; $1.8 \%$ from vaginal samples. Practitioners were instructed to obtain the sample from the transformation zone of the cervix using a Rovers ${ }^{\circledR}$ Cervex Brush. Swabs were placed in SurePath ${ }^{\circledR}$ media. TriPath Imaging Inc. equipment was used to process samples according to the manufacturer's instructions. Cervical smears were interpreted by Canadian-registered CCSP cytotechnologists and the BC Cancer Agency-based cytopathologists. Cytological interpretation was reported using the British Society of Clinical Cytology terminology currently in use in BC. For this analysis, however, results were reclassified using the Bethesda system. Negative and benign changes were kept as originally categorized. Mild dyskariosis was classified as low-grade intraepithelial lesions (LGIL) of the squamous or glandular type; moderate or severe dyskariosis and suspicious smears were classified as high-grade intraepithelial lesions (HGIL) of the squamous or glandular type. Smears showing squamous $(87.7 \%)$ and glandular $(12.3 \%)$ abnormalities were not separated in our main analysis of LGIL or HGIL for simplicity of data presentation. Individual typing data has been separated by glandular or squamous type and is included in a separate table (Table 1). The categories of ASCUS and AGUS were not used.

This study was approved by the joint Clinical Research Ethics Board of the BC Cancer Agency and the University of British Columbia. Use of specimens for this study was performed according to the 'Secondary Use of Personal Information in Health Research: Case Studies' (Canadian Institutes of Health Research, November 2002). Cytology results were recorded in the CCSP database. Each sample was assigned a study number, and the data including the age of the participant, geographic region of the smear taker, cytology result and previous screening history were attached to the study number. Subsequently, the remainder of each sample and the data were stripped of potential patient identifiers. The data and samples left over after cytology were then transferred to the Genome Sciences Centre at the BC Cancer Research Centre for HPV analysis.

\section{Study sample selection}

From the total study sample set of 8,700 , forty samples were from repeat smears from the same women and were excluded, leaving 8,660 independent samples. PCR analysis was performed on 4,980 samples including all 614 cytologically abnormal samples and a random selection (every second sample by study number) of 4,366 normal and benign cytology smears. This sample showed a representative distribution to that of the remaining samples. Neither normal nor benign smears showed a statistically significant difference in age distribution or geographic location between selected and not selected smears. Age was tested using the $t$-test, and also using Mantel-Hanzel chi-square analysis with six age categories $(<20,20-29$, $30-39,40-49,50-59$ and 60+). Geographic location was tested using the chi-square test.

DNA extraction, quantification and quality control

The portion of each sample remaining after cytology (1$6 \mathrm{ml}$ ) was pelleted by centrifugation, re-suspended in $300 \mu \mathrm{l}$ of phosphate-buffered saline, and stored at $-80^{\circ} \mathrm{C}$. DNA was extracted from $150 \mu \mathrm{l}$ of thawed re-suspended cellular material using the PureGene DNA isolation kit (Gentra Systems, MN, USA). DNA samples were quantified by fluorometry and $10 \mathrm{ng}$ aliquots arrayed in 96-well plates for PCR analysis. Plates were arrayed according to sample number and were not separated according to 
Table 1 HPV type distribution according to cellular origin of abnormality, 95\% CI shown in brackets

Cell type could not be determined for three patients. Their results are not shown in the table

${ }^{\text {a }}$ HPV of unidentified type

\begin{tabular}{|c|c|c|c|c|}
\hline & LGIL squamous (\%) & LGIL adeno. (\%) & HGIL squamous (\%) & HGIL adeno (\%) \\
\hline Sample no. & 441 & 61 & 52 & 9 \\
\hline $\mathrm{HPV}+$ & $70.3(65.8,74.5)$ & $62.3(48.9,74.1)$ & $80.8(67.0,89.9)$ & $77.8(40.2,96.1)$ \\
\hline Any HR type & $53.1(48.3,57.8)$ & $45.9(33.3,59.1)$ & $78.8(64.9,88.5)$ & $77.8(40.2,96.1)$ \\
\hline 16 or 18 & $36.5(32.0,41.2)$ & $45.9(33.3,59.1)$ & $61.5(47.0,74.4)$ & $66.7(30.9,91.0)$ \\
\hline 16 & $33.8(29.4,38.4)$ & $44.3(31.8,57.5)$ & $51.9(37.8,65.8)$ & $55.6(22.7,84.7)$ \\
\hline 18 & $5.4(3.6,8.1)$ & $9.8(4.1,20.9)$ & $23.1(13.0,37.2)$ & $11.1(0.6,49.3)$ \\
\hline 26 & $0.0(0.0,1.1)$ & $0.0(0.0,7.4)$ & $0.0(0.0,8.6)$ & $0.0(0.0,37.1)$ \\
\hline 31 & $2.7(1.5,4.8)$ & $0.0(0.0,7.4)$ & $3.8(0.7,14.3)$ & $0.0(0.0,37.1)$ \\
\hline 33 & $5.2(3.4,7.8)$ & $4.9(1.3,14.6)$ & $7.7(2.5,19.4)$ & $0.0(0.0,37.1)$ \\
\hline 35 & $5.2(3.4,7.8)$ & $6.6(2.1,16.7)$ & $3.8(0.7,14.3)$ & $0.0(0.0,37.1)$ \\
\hline 39 & $0.2(0.0,1.5)$ & $0.0(0.0,7.4)$ & $0.0(0.0,8.6)$ & $0.0(0.0,37.1)$ \\
\hline 45 & $1.4(0.6,3.1)$ & $0.0(0.0,7.4)$ & $0.0(0.0,8.6)$ & $0.0(0.0,37.1)$ \\
\hline 51 & $0.0(0.0,1.1)$ & $0.0(0.0,7.4)$ & $0.0(0.0,8.6)$ & $0.0(0.0,37.1)$ \\
\hline 52 & $0.2(0.0,1.5)$ & $1.6(0.1,10.0)$ & $3.8(0.7,14.3)$ & $0.0(0.0,37.1)$ \\
\hline 53 & $0.0(0.0,1.1)$ & $1.6(0.1,10.0)$ & $0.0(0.0,8.6)$ & $0.0(0.0,37.1)$ \\
\hline 56 & $7.0(4.9,9.9)$ & $6.6(2.1,16.7)$ & $1.9(0.1,11.6)$ & $0.0(0.0,37.1)$ \\
\hline 58 & $2.3(1.2,4.3)$ & $1.6(0.1,10.0)$ & $7.7(2.5,19.4)$ & $0.0(0.0,37.1)$ \\
\hline 59 & $0.7(0.2,2.1)$ & $1.6(0.1,10.0)$ & $3.8(0.7,14.3)$ & $0.0(0.0,37.1)$ \\
\hline 66 & $2.5(1.3,4.6)$ & $0.0(0.0,7.4)$ & $0.0(0.0,8.6)$ & $11.1(0.6,49.3)$ \\
\hline 68 & $0.2(0.0,1.5)$ & $0.0(0.0,7.4)$ & $0.0(0.0,8.6)$ & $0.0(0.0,37.1)$ \\
\hline 73 & $0.7(0.2,2.1)$ & $0.0(0.0,7.4)$ & $1.9(0.1,11.6)$ & $0.0(0.0,37.1)$ \\
\hline 82 & $0.2(0.0,1.5)$ & $0.0(0.0,7.4)$ & $0.0(0.0,8.6)$ & $0.0(0.0,37.1)$ \\
\hline Any LR type & $25.2(21.2,29.5)$ & $23.0(13.5,35.8)$ & $5.8(1.5,16.9)$ & $11.1(0.6,49.3)$ \\
\hline 6 or 11 & $15.2(12.0,19.0)$ & $18.0(9.8,30.4)$ & $3.8(0.7,14.3)$ & $11.1(0.6,49.3)$ \\
\hline 6 & $14.7(11.6,18.5)$ & $14.8(7.4,26.7)$ & $3.8(0.7,14.3)$ & $11.1(0.6,49.3)$ \\
\hline 11 & $0.9(0.3,2.5)$ & $4.9(1.3,14.6)$ & $0.0(0.0,8.6)$ & $0.0(0.0,37.1)$ \\
\hline 30 & $0.7(0.2,2.1)$ & $0.0(0.0,7.4)$ & $0.0(0.0,8.6)$ & $0.0(0.0,37.1)$ \\
\hline 42 & $1.6(0.7,3.4)$ & $0.0(0.0,7.4)$ & $0.0(0.0,8.6)$ & $0.0(0.0,37.1)$ \\
\hline 43 & $0.2(0.0,1.5)$ & $0.0(0.0,7.4)$ & $1.9(0.1,11.6)$ & $0.0(0.0,37.1)$ \\
\hline 44 & $0.0(0.0,1.1)$ & $0.0(0.0,7.4)$ & $0.0(0.0,8.6)$ & $0.0(0.0,37.1)$ \\
\hline 54 & $1.1(0.4,2.8)$ & $0.0(0.0,7.4)$ & $0.0(0.0,8.6)$ & $0.0(0.0,37.1)$ \\
\hline 61 & $0.0(0.0,1.1)$ & $0.0(0.0,7.4)$ & $0.0(0.0,8.6)$ & $0.0(0.0,37.1)$ \\
\hline 63 & $0.0(0.0,1.1)$ & $0.0(0.0,7.4)$ & $0.0(0.0,8.6)$ & $0.0(0.0,37.1)$ \\
\hline 67 & $1.8(0.8,3.7)$ & $1.6(0.1,10.0)$ & $0.0(0.0,8.6)$ & $0.0(0.0,37.1)$ \\
\hline 69 & $0.0(0.0,1.1)$ & $0.0(0.0,7.4)$ & $0.0(0.0,8.6)$ & $0.0(0.0,37.1)$ \\
\hline 70 & $0.5(0.1,1.8)$ & $0.0(0.0,7.4)$ & $0.0(0.0,8.6)$ & $0.0(0.0,37.1)$ \\
\hline 72 & $0.7(0.2,2.1)$ & $4.9(1.3,14.6)$ & $0.0(0.0,8.6)$ & $0.0(0.0,37.1)$ \\
\hline 74 & $0.2(0.0,1.5)$ & $0.0(0.0,7.4)$ & $0.0(0.0,8.6)$ & $0.0(0.0,37.1)$ \\
\hline 81 & $0.9(0.3,2.5)$ & $0.0(0.0,7.4)$ & $0.0(0.0,8.6)$ & $0.0(0.0,37.1)$ \\
\hline 83 & $0.0(0.0,1.1)$ & $0.0(0.0,7.4)$ & $0.0(0.0,8.6)$ & $0.0(0.0,37.1)$ \\
\hline 84 & $0.2(0.0,1.5)$ & $0.0(0.0,7.4)$ & $0.0(0.0,8.6)$ & $0.0(0.0,37.1)$ \\
\hline 87 & $0.5(0.1,1.8)$ & $0.0(0.0,7.4)$ & $1.9(0.1,11.6)$ & $0.0(0.0,37.1)$ \\
\hline 89 & $0.0(0.0,1.1)$ & $0.0(0.0,7.4)$ & $0.0(0.0,8.6)$ & $0.0(0.0,37.1)$ \\
\hline 90 & $3.6(2.2,5.9)$ & $3.3(0.6,12.4)$ & $1.9(0.1,11.6)$ & $0.0(0.0,37.1)$ \\
\hline HPV $X^{a}$ & $1.4(0.6,3.1)$ & $4.9(1.3,14.6)$ & $0.0(0.0,8.6)$ & $0.0(0.0,37.1)$ \\
\hline
\end{tabular}

cytology. The $\beta$-globin gene primers were used to confirm the competence each DNA sample to support PCR. The percentage of samples that passed this quality control test is
$96.8 \%(4,821$ samples) samples that did not pass this test were not included in HPV testing (see Supplemental Online Figure A). 
HPV testing and HPV type determination

Tagged GP5+/GP6+ consensus primers [9, 10] were used to detect HPV by amplifying a $150 \mathrm{bp}$ sequence of the viral L1 gene from virtually any HPV type, and bi-directional sequencing was used to determine HPV type(s) present in each sample. The GP5+/6+ primers $[9,10]$ were modified by the addition of SeqA2 (GAATTCTCTAGATGATCA GCGGC) or Seq B2 (CGAACTTTATTCGGTCGAAAA GG) tags to their $5^{\prime}$ ends to simplify later sequencing. Testing of known HPV types mixed with genomic DNA demonstrated effectiveness of the tagged primers in detecting various HPV types. PCR analysis was carried out as previously described [9] with minimal changes $\left(95^{\circ} \mathrm{C}\right.$ $30 \mathrm{~s}, 40^{\circ} \mathrm{C} 1 \mathrm{~min}, 68^{\circ} \mathrm{C} 30 \mathrm{~s}$ for 40 cycles). An aliquot of each PCR product was separated on a $3 \%$ agarose gel for visualization. Samples that showed the expected $150 \mathrm{bp}$ band were designated as HPV positive. Aliquots of PCR products from HPV positive samples were then re-arrayed into 96-well plates and purified by the AMPure magnetic bead system (Agencourt Bioscience Corporation, Beverly, Massachusetts, USA). Purified PCR products were bidirectionally sequenced using BigDye 3.1 at 1/24 chemistry and run on $3730 x 1$ capillary sequencers (Applied Biosystems, Foster City, California). Sequence traces that produced apparent multiple overlapping sequences were flagged as possible multiple infections (MI). PCR products from such samples were phosphorylated with polynucleotide kinase (New England BioLabs, MA, USA) and subcloned by blunt end ligation into pUC19. Sixteen clones of each putative MI were bi-directionally sequenced using the -21 M13 Forward (TGTAAAACGACGGCCAGT) and M13 Reverse (CAGGAAACAGCTATGAC) primers. Sequences were aligned to a database of all known HPV L1 sequences using local BLAST alignment, and the best match scored as a specific HPV type if it had greater than $95 \%$ similarity over more than 50 bases.

For this study types $16,18,26,31,33,35,39,45,51,52$, $53,56,58,59,66,68,73$, and 82 were considered high-risk HPV types.

\section{Statistical analyses}

Statistical analyses were performed using the SAS package (SAS Institute Inc., Cary, NC, USA). All cytologically abnormal samples were HPV typed, but not all normal or benign samples were typed; it was, therefore, necessary to weight by cytology in the final prevalence analyses. Weighting was performed as follows. Column "Study Sample" in Tables 2 and 3 adjusts prevalence estimated from successful HPV testing to reflect cytology distribution in the study sample. The weight for each normal, benign, LGIL and HGIL is the proportion it constitutes of the study sample, divided by the proportion it constitutes of successful HPV tests. Multiply infected samples were defined as samples for which two or more HPV types were detected. Such samples were counted as a positive for one type of HPV and also included among positives for another or other types of HPV, in calculations of the prevalence of each HPV type.

The Cochrane-Armitage trend test was used on HPV prevalence rates by 5 -year age groups shown in Figs. 1 and 2 a.

\section{Results}

Study participants are representative of the CCSP in terms of age, cytology and geographical distribution

Table 4 shows the age distribution of the study sample set compared to that of the entire CCSP in 2004, including 95\% CI. While the difference in some categories is statistically significant, differences are small in practical terms. Furthermore, adjustment by age, by cytology or both has been included among HPV prevalence estimates. The age distribution is comparable, except that the study sample shows modest over-recruitment of women in the two youngest ( $<20$ and $20-24$ year old) age groups. The distribution of cytology is similar to that of the CCSP (Table 4). Of the 8,660 independent samples, 9 were unsatisfactory for interpretation. Of the interpretable samples, 65 showed high-grade intraepithelial lesions (HGIL, $0.8 \%), 549$ showed low-grade intraepithelial lesions (LGIL, 6.3\%), 413 showed benign changes (4.8\%), and the remaining 7,624 were cytologically normal (88.1\%) [8]. Table 4 also shows that the study sample is distributed, by health authority region, comparably to the CCSP.

Prevalence and type distribution

Table 2 summarizes both the overall HPV prevalence and type distribution by cytology, with $95 \%$ confidence intervals. Figure 1 illustrates these data graphically. The overall HPV prevalence of the study population, adjusted from the data for the 4,980 tested samples as described earlier, was $16.8 \%$. Of them $13.9 \%$ were positive for high risk HPV, and $11.6 \%$ had the high risk types 16 or 18 that are targeted by vaccines. HPV prevalence increases with each moresevere cytological category. HPV16 is the most common type, found in $10.7 \%$ of samples. The HPV16 prevalence generally increases with the severity of the abnormalities that are precursors to cervical cancer; it is present in $8.7 \%$ of cytologically normal samples, $35.2 \%$ of LGIL and $52.4 \%$ of HGIL. Age-adjusted data can be found in Supplemental Online Table A. Adjusting for age slightly reduces the prevalence of HPV overall (to 15.5\%) and in 
Table 2 HPV Prevalence and type distribution, shown by cytology group, (95\% CI shown in parentheses)

\begin{tabular}{|c|c|c|c|c|c|}
\hline & Study sample ${ }^{\mathrm{a}}$ & Normal & Benign & LGIL & HGIL \\
\hline Sample no. & 4,821 & 4,003 & 250 & 505 & 63 \\
\hline $\mathrm{HPV}+$ & $16.8(15.8,17.9)$ & $12.3(11.3,13.4)$ & $19.6(15.0,25.2)$ & $69.3(65.0,73.3)$ & $81.0(68.7,89.4)$ \\
\hline Any HR type & $13.9(13.0,14.9)$ & $10.6(9.6,11.6)$ & $14.4(10.4,19.5)$ & $52.3(47.8,56.7)$ & $79.4(67.0,88.1)$ \\
\hline 16 or 18 & $11.6(10.8,12.6)$ & $9.3(8.5,10.3)$ & $10.8(7.4,15.5)$ & $37.8(33.6,42.2)$ & $63.5(50.4,75.0)$ \\
\hline 16 & $10.7(9.8,11.6)$ & $8.7(7.9,9.6)$ & $7.6(4.8,11.8)$ & $35.2(31.1,39.6)$ & $52.4(39.5,65.0)$ \\
\hline 18 & $3.5(3.1,4.1)$ & $3.1(2.6,3.7)$ & $5.6(3.2,9.4)$ & $5.9(4.1,8.5)$ & $22.2(13.1,34.8)$ \\
\hline 26 & $0.0(0.0,0.1)$ & $0.0(0.0,0.1)$ & $0.0(0.0,1.9)$ & $0.0(0.0,0.9)$ & $0.0(0.0,7.2)$ \\
\hline 31 & $0.4(0.3,0.7)$ & $0.2(0.1,0.4)$ & $0.8(0.1,3.2)$ & $2.4(1.3,4.2)$ & $3.2(0.6,12.0)$ \\
\hline 33 & $1.1(0.9,1.5)$ & $0.8(0.6,1.2)$ & $0.4(0.0,2.6)$ & $5.1(3.5,7.6)$ & $6.3(2.1,16.3)$ \\
\hline 35 & $0.9(0.6,1.2)$ & $0.5(0.3,0.8)$ & $0.4(0.0,2.6)$ & $5.3(3.6,7.8)$ & $3.2(0.6,12.0)$ \\
\hline 39 & $0.0(0.0,0.1)$ & $0.0(0.0,0.1)$ & $0.0(0.0,1.9)$ & $0.2(0.0,1.3)$ & $0.0(0.0,7.2)$ \\
\hline 45 & $0.6(0.4,0.9)$ & $0.6(0.4,0.9)$ & $0.0(0.0,1.9)$ & $1.2(0.5,2.7)$ & $0.0(0.0,7.2)$ \\
\hline 51 & $0.0(0.0,0.1)$ & $0.0(0.0,0.1)$ & $0.0(0.0,1.9)$ & $0.0(0.0,0.9)$ & $0.0(0.0,7.2)$ \\
\hline 52 & $0.1(0.0,0.2)$ & $0.0(0.0,0.2)$ & $0.0(0.0,1.9)$ & $0.4(0.1,1.6)$ & $3.2(0.6,12.0)$ \\
\hline 53 & $0.1(0.0,0.2)$ & $0.0(0.0,0.2)$ & $0.4(0.0,2.6)$ & $0.2(0.0,1.3)$ & $0.0(0.0,7.2)$ \\
\hline 56 & $2.0(1.6,2.4)$ & $1.6(1.3,2.1)$ & $2.0(0.7,4.9)$ & $6.9(4.9,9.6)$ & $1.6(0.1,9.7)$ \\
\hline 58 & $0.7(0.5,1.0)$ & $0.6(0.4,0.9)$ & $0.0(0.0,1.9)$ & $2.2(1.1,4.0)$ & $6.3(2.1,16.3)$ \\
\hline 59 & $0.5(0.3,0.7)$ & $0.4(0.2,0.7)$ & $0.8(0.1,3.2)$ & $0.8(0.3,2.2)$ & $3.2(0.6,12.0)$ \\
\hline 66 & $0.9(0.7,1.2)$ & $0.8(0.6,1.1)$ & $1.2(0.3,3.8)$ & $2.2(1.1,4.0)$ & $1.6(0.1,9.7)$ \\
\hline 68 & $0.0(0.0,0.1)$ & $0.0(0.0,0.1)$ & $0.0(0.0,1.9)$ & $0.2(0.0,1.3)$ & $0.0(0.0,7.2)$ \\
\hline 73 & $0.2(0.1,0.4)$ & $0.1(0.0,0.3)$ & $0.4(0.0,2.6)$ & $0.6(0.2,1.9)$ & $1.6(0.1,9.7)$ \\
\hline 82 & $0.0(0.0,0.1)$ & $0.0(0.0,0.1)$ & $0.0(0.0,1.9)$ & $0.2(0.0,1.3)$ & $0.0(0.0,7.2)$ \\
\hline Any LR type & $7.0(6.3,7.7)$ & $5.6(4.9,6.3)$ & $8.8(5.7,13.2)$ & $25.0(21.3,29.0)$ & $6.3(2.1,16.3)$ \\
\hline 6 or 11 & $4.2(3.7,4.8)$ & $3.3(2.8,3.9)$ & $5.6(3.2,9.4)$ & $15.6(12.6,19.2)$ & $4.8(1.2,14.2)$ \\
\hline 6 & $4.0(3.5,4.6)$ & $3.2(2.7,3.8)$ & $5.2(2.9,8.9)$ & $14.9(11.9,18.3)$ & $4.8(1.2,14.2)$ \\
\hline 11 & $0.2(0.1,0.4)$ & $0.1(0.0,0.3)$ & $0.4(0.0,2.6)$ & $1.4(0.6,3.0)$ & $0.0(0.0,7.2)$ \\
\hline 30 & $0.2(0.1,0.3)$ & $0.1(0.0,0.3)$ & $0.4(0.0,2.6)$ & $0.6(0.2,1.9)$ & $0.0(0.0,7.2)$ \\
\hline 42 & $0.2(0.1,0.4)$ & $0.1(0.1,0.3)$ & $0.0(0.0,1.9)$ & $1.4(0.6,3.0)$ & $0.0(0.0,7.2)$ \\
\hline 43 & $0.3(0.2,0.5)$ & $0.3(0.2,0.6)$ & $0.0(0.0,1.9)$ & $0.2(0.0,1.3)$ & $1.6(0.1,9.7)$ \\
\hline 44 & $0.0(0.0,0.1)$ & $0.0(0.0,0.2)$ & $0.0(0.0,1.9)$ & $0.0(0.0,0.9)$ & $0.0(0.0,7.2)$ \\
\hline 54 & $0.5(0.3,0.8)$ & $0.5(0.3,0.8)$ & $0.4(0.0,2.6)$ & $1.0(0.4,2.4)$ & $0.0(0.0,7.2)$ \\
\hline 61 & $0.0(0.0,0.2)$ & $0.0(0.0,0.2)$ & $0.4(0.0,2.6)$ & $0.0(0.0,0.9)$ & $0.0(0.0,7.2)$ \\
\hline 63 & $0.0(0.0,0.1)$ & $0.0(0.0,0.2)$ & $0.0(0.0,1.9)$ & $0.0(0.0,0.9)$ & $0.0(0.0,7.2)$ \\
\hline 67 & $0.6(0.4,0.9)$ & $0.6(0.4,0.9)$ & $0.4(0.0,2.6)$ & $1.8(0.9,3.5)$ & $0.0(0.0,7.2)$ \\
\hline 69 & $0.0(0.0,0.1)$ & $0.0(0.0,0.2)$ & $0.0(0.0,1.9)$ & $0.0(0.0,0.9)$ & $0.0(0.0,7.2)$ \\
\hline 70 & $0.2(0.1,0.4)$ & $0.2(0.1,0.4)$ & $0.0(0.0,1.9)$ & $0.4(0.1,1.6)$ & $0.0(0.0,7.2)$ \\
\hline 72 & $0.2(0.1,0.4)$ & $0.1(0.0,0.3)$ & $0.0(0.0,1.9)$ & $1.2(0.5,2.7)$ & $0.0(0.0,7.2)$ \\
\hline 74 & $0.1(0.0,0.2)$ & $0.1(0.0,0.2)$ & $0.0(0.0,1.9)$ & $0.2(0.0,1.3)$ & $0.0(0.0,7.2)$ \\
\hline 81 & $0.3(0.2,0.5)$ & $0.2(0.1,0.4)$ & $0.8(0.1,3.2)$ & $0.8(0.3,2.2)$ & $0.0(0.0,7.2)$ \\
\hline 83 & $0.0(0.0,0.1)$ & $0.0(0.0,0.1)$ & $0.4(0.0,2.6)$ & $0.0(0.0,0.9)$ & $0.0(0.0,7.2)$ \\
\hline 84 & $0.1(0.0,0.2)$ & $0.1(0.0,0.2)$ & $0.0(0.0,1.9)$ & $0.2(0.0,1.3)$ & $0.0(0.0,7.2)$ \\
\hline 87 & $0.1(0.1,0.3)$ & $0.1(0.0,0.3)$ & $0.0(0.0,1.9)$ & $0.4(0.1,1.6)$ & $1.6(0.1,9.7)$ \\
\hline 89 & $0.0(0.0,0.1)$ & $0.0(0.0,0.2)$ & $0.0(0.0,1.9)$ & $0.0(0.0,0.9)$ & $0.0(0.0,7.2)$ \\
\hline 90 & $1.4(1.1,1.8)$ & $1.2(0.9,1.6)$ & $2.4(1.0,5.4)$ & $3.6(2.2,5.7)$ & $1.6(0.1,9.7)$ \\
\hline HPV $X^{b}$ & $0.2(0.1,0.3)$ & $0.0(0.0,0.2)$ & $0.4(0.0,2.6)$ & $1.8(0.9,3.5)$ & $0.0(0.0,7.2)$ \\
\hline
\end{tabular}

${ }^{a}$ Weighting in this column is by cytology

b HPV of unidentified type 
Table 3 Multiple infections, shown by cytology group (95\% CI shown in parentheses)

\begin{tabular}{lccccc}
\hline & Study Sample $^{\mathrm{a}}$ & Normal & Benign & LGIL & HGIL \\
\hline Sample no. & 4,821 & 4,003 & 250 & 505 & 63 \\
HPV+ & $16.8(15.8,17.9)$ & $12.3(11.3,13.4)$ & $19.6(15.0,25.2)$ & $69.3(65.0,73.3)$ & $81.0(68.7,89.4)$ \\
MI rate & $5.5(4.9,6.2)$ & $4.9(4.3,5.6)$ & $4.3(2.2,8.1)$ & $13.7(10.2,18.2)$ & $13.9(5.2,30.3)$ \\
MI any HR & $5.4(4.8,6.1)$ & $4.8(4.2,5.5)$ & $4.3(2.2,8.1)$ & $13.4(9.9,17.9)$ & $13.9(5.2,30.3)$ \\
MI any LR & $4.3(3.7,4.9)$ & $3.9(3.4,4.5)$ & $3.9(1.9,7.5)$ & $10.1(7.1,14.2)$ & $5.6(1.0,20.0)$ \\
MI any HR with any LR & $4.3(3.7,4.9)$ & $3.9(3.3,4.5)$ & $3.9(1.9,7.5)$ & $9.8(6.8,13.8)$ & $5.6(1.0,20.0)$ \\
16 MI & $4.7(4.1,5.3)$ & $4.3(3.7,5.0)$ & $3.0(1.3,6.4)$ & $10.5(7.4,14.6)$ & $11.1(3.6,27.0)$ \\
18 MI & $2.9(2.5,3.5)$ & $2.7(2.3,3.3)$ & $3.5(1.6,7.0)$ & $4.2(2.4,7.3)$ & $13.9(5.2,30.3)$ \\
6 MI & $3.3(2.8,3.8)$ & $2.9(2.4,3.5)$ & $3.5(1.6,7.0)$ & $8.2(5.5,12.0)$ & $5.6(1.0,20.0)$ \\
\hline
\end{tabular}

${ }^{\text {a }}$ Values in this column are weighted by cytology

Fig. 1 Prevalence of individual HPV types and categories by cytology. Data are not adjusted for ages. 95\% confidence intervals are shown

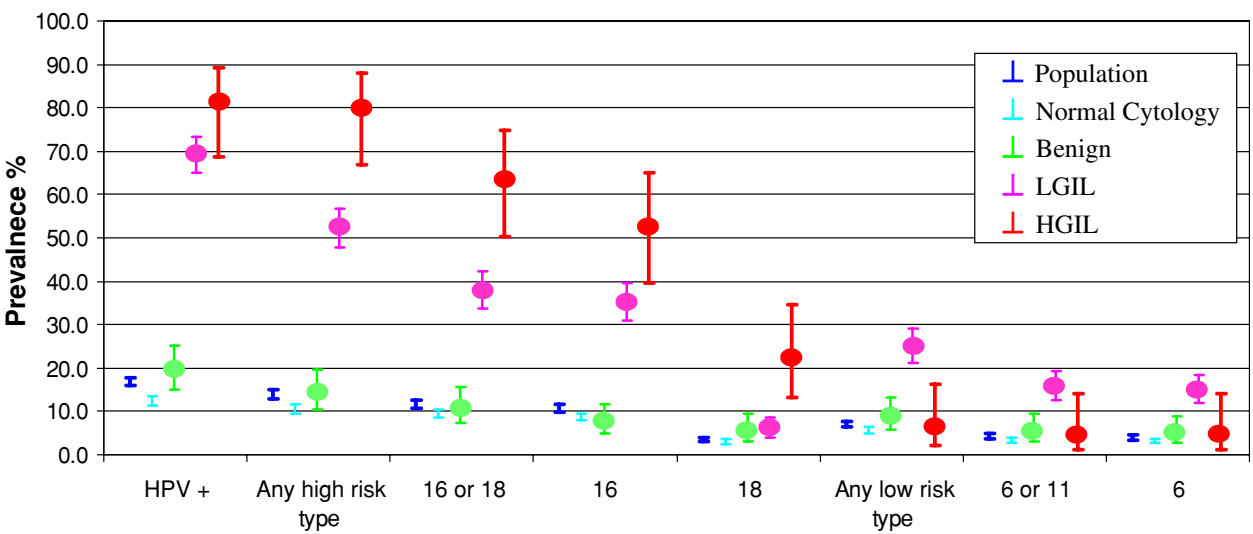

severity of the lesions (from $39.8 \%$ in normal samples to $19.8 \%$ in LGIL and $17.2 \%$ in HGIL). HGIL have a higher percentage of MI only because they have more HPV; the HPV infections they have are more likely to be single HPV types.

\section{Discussion}

This study provides an estimate of the prevalence of HPV in women participating in routine cytology screening in BC. This is the largest typing study of its kind in Canada to date and one of the largest single-center studies worldwide. While the HPV prevalence of screened women is not necessarily equivalent to that of all women [11] in BC, the high participation rate of the CCSP (70\% [12]) argues that it provides a good estimate for the province. The use of direct sequencing theoretically allows the detection of all known HPV types and provides a level of detail not attainable using existing hybridization probe sets or the Digene Hybrid Capture 2 system.

Several international studies have examined the prevalence of HPV in women. The diversity of population samples, sample media and HPV typing methods make it difficult to identify studies that are exactly comparable to 
Fig. 2 HPV prevalence by 5year age strata. $95 \%$ Confidence intervals are shown. In BC, cervical cancer screening is not recommended for women over 70 , so those over 70 in this sample represent a nonrandom set of women who likely presented with symptoms and for whom cytology was conducted as part of a diagnostic work-up
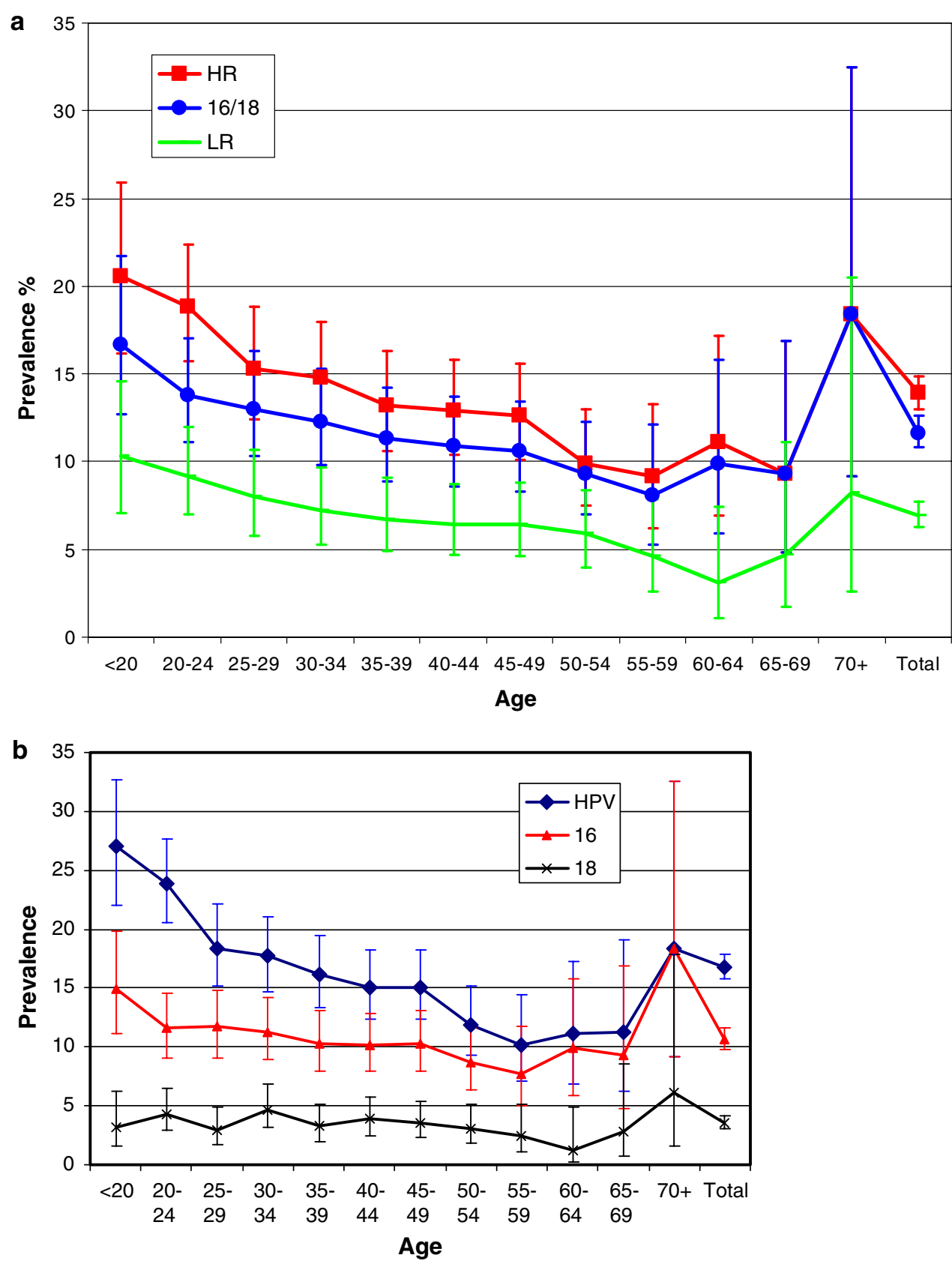

each other. It is not surprising that our HPV type distribution differs from that of a large US study based on selfsampled vaginal swabs [13]. Low-risk HPV types that are more prevalent in the vagina and vulva [14] will not be well represented in our samples, as these are almost exclusively cervical smears. It is also reported that selfcollected vaginal sampling methods are generally less sensitive than cervical smears for the detection of HPV [15, 16]. Overall HPV infection rates in population-based studies where all women were included found HPV prevalence rates from $2 \%$ in Hanoi, Vietnam [17], to $40 \%$ in Mozambique [18]. Our overall HPV infection rate was $16.8 \%$, close to that of an Ontario study (13.3\%) [19]. A recent large study in The Netherlands typing high-risk HPV in the population found a rate of $5.6 \%$ [20] and shows a similar trend for age as observed in our study (Fig. 2a, b). Our prevalence is also at a similar level to that seen in a recent, large meta-analysis for Asian women at $14.4 \%$ for cytologically normal samples [21]. Prevalence of HR HPV ranged from $4.4 \%$ [22] to almost $20 \%$ [23] in these studies, in keeping with our rate of $13.9 \%$. Similarly to many other studies, HPV16 was the most common high-risk type in BC. We found a higher prevalence of HPV16 (10.7\%) than other studies, which showed less than $1 \%$ to just over $5 \%$. Several studies $[17,22-28]$ used GP5+/6+ primers, but detected HPV types by hybridization followed by 
Table 4 Comparison of study sample composition to the Cervical Cancer screening program in BC, data for 2004

\begin{tabular}{lcc}
\hline & $\begin{array}{l}\text { Study sample } \\
(\% \text { and } 95 \% \text { CI })\end{array}$ & $\begin{array}{c}\text { CCSP } \\
\text { Cytology }\end{array}$ \\
$\quad$ Normal & & \\
Benign & $88.1(87.4,88.8)$ & 89.9 \\
LGIL & $4.8(4.3,5.2)$ & 3.1 \\
HGIL & $6.3(5.8,6.9)$ & 5.9 \\
Region of BC & $0.8(0.6,0.9)$ & 1.1 \\
Vancouver coastal & $60.3(59.3,61.4)$ & 60.2 \\
Fraser & $18.5(17.7,19.3)$ & 20.6 \\
Interior & $9.4(8.7,10.0)$ & 7.5 \\
Northern & $7.5(7.0,8.1)$ & 7.0 \\
Vancouver Island & $4.3(3.8,4.7)$ & 4.3 \\
Age & & \\
$<20$ & $5.4(4.9,5.9)$ & 4.7 \\
$20-24$ & $11.2(10.5,11.9)$ & 9.5 \\
$25-29$ & $10.5(9.8,11.1)$ & 11.0 \\
$30-34$ & $12.2(11.5,12.9)$ & 12.8 \\
$35-39$ & $12.0(11.3,12.7)$ & 12.8 \\
$40-44$ & $12.6(11.9,13.3)$ & 13.3 \\
$45-49$ & $12.5(11.8,13.2)$ & 11.8 \\
$50-54$ & $10.2(9.6,10.9)$ & 9.2 \\
$55-59$ & $6.5(6.0,7.0)$ & 6.5 \\
$60-64$ & $3.5(3.1,3.8)$ & 4.2 \\
$65-69$ & $2.3(2.0,2.6)$ & 2.8 \\
$70+$ & $1.1(0.8,1.3)$ & 1.5 \\
\hline
\end{tabular}

Table 4 details a comparison of the study sample to the 2004 CCSP. Distribution by cytology, health region in BC (location where smear was taken), and age by 5 year strata. $95 \%$ confidence intervals are shown in brackets

enzyme-based immunoassay. Our method subjects the PCR products to an additional, albeit linear, amplification in the sequencing reaction, likely enhancing the sensitivity of detection. Comparison of cycle sequencing, line blotting and hybrid capture showed that sequencing is the most sensitive [29].

HPV positivity increases, as expected, from normal (12.3\%), to benign (19.6\%), to LGIL (69.3\%), to HGIL (81.0\%). The trend for HPV 16 also makes sense, going from normal $(8.7 \%)$, benign $(7.6 \%)$, LGIL $(35.2 \%)$ and HGIL (52.4\%). The relative proportion of HPV16 (HPV16/ totalHPV), however, is unexpectedly high in normal samples $(71 \%)$, when compared to benign (39\%), LGIL (51\%) and HGIL $(65 \%)$. It is unlikely that contamination could account for this difference, because all samples were processed on multi-well plates and were not separated according to cytology. We propose that there is a real biological explanation for this observation that likely relates to the sensitivity of PCR and sequencing to detect HPV. We may be detecting transient, sub-clinical HPV exposures in addition to overt HPV16 infections that would be detected with less sensitive techniques.

We found that $33 \%$ of HPV positive samples contained multiple HPV types, within the range of $12-62 \%$ seen in other studies $[28,30]$. Direct sequencing may underestimate the MI rate; however, our conservative over selection of potential MIs (all sequence traces with any sign of mixed types were subcloned) should compensate for this. Our higher observed prevalence of HPV16 is not likely to be a result of our intensive characterization of MI samples.

The percentage of HPV positive samples that had multiple infections was higher in the cytologically normal HPV positive samples, possibly reflecting clonal outgrowth of cells infected with a single HPV type in the pre-cancerous lesions. This may imply that multiple types of HPV simultaneously infect the same woman but not necessarily the same individual cells, or it may reflect the preferential persistence of one HPV type. Multiple infections may be more recent infections that have had less time for one or some of the types involved to be cleared.

We did not exclude women who were tested as a followup to a previous abnormal smear. The smear-takers were high-volume sites; this could bias toward young sexually active women who are seeking birth control. Compared to the CCSP in 2004, our sample has a higher proportion of young women, who would be more likely to have current HPV infections. Sellors and colleagues showed a lower rate ( $9.6 \%$ for high-risk HPV) in older women than in younger women [31]; we also observe this trend. Thus, our study could slightly over estimate the prevalence of HPV infection relative to the general female population of $\mathrm{BC}$.

Differences between recruitment methods can complicate direct comparison of our findings to those of other Canadian studies [11, 19, 32, 33]. An Ontario study [19] using Digene Hybrid Capture 2 and PCR showed a prevalence range of 25-6\% depending on age; Montreal University students had an HPV rate of $29 \%$ [33], similar to the prevalence we observed in this age group. A recent international analysis by IARC [28] illustrates the differences in type distribution in different countries. We detect HPV90, but this type was not included in the probe set used by IARC [28]. Types seen more commonly in Asian countries (such as 51, 52 and 58) were not significantly increased in BC, despite its large Asian population. Comparison to worldwide data [28] demonstrates our ability to detect most or all known HPV types, despite the tendency of GP5+/6+ primer set to underestimate HPV 52 [34].

Prophylactic vaccines are now available against HR HPV types 16 and 18. Efficacy evaluations to date for these vaccines show $100 \%$ protection against development of LGIL and HGIL associated with the HPV types targeted. A 
minimum estimate of the impact of a vaccine protecting against HPV16 and HPV18 would be the proportion of a lesion for which representative samples are positive for 16 , 18 , or 16 and 18, but not other HR types of HPV. In our data, the proportion of LGIL and HGIL samples that meet this criterion is $29.9 \%$ for LGIL and $55.6 \%$ for HGIL (data not shown). Conservatively, we predict that vaccinating against HPV16 and 18 would, in an effectively vaccinated group, prevent the development of one-third of LGIL and more than half of HGIL, and an even larger proportion of cervical cancer. If these estimates are expressed as a percentage of those LGIL and HGIL that had detectable HR HPV, a likely more realistic estimate (57.2\% of LGIL and $70.0 \%$ of HGIL) of the percentage of these lesions that are attributable to vaccine-related HR types is obtained. Including additional HPV types in future vaccines (such as 56 and 90 in BC) would further increase the percentage of cervical lesions prevented. These data provide a baseline from which to monitor changes in HPV prevalence that result from future use of HPV vaccines in BC and may inform the use of HPV testing as a first-line screening alternative to cytology.

Acknowledgments We thank Jerry Liu for assistance with computational analysis of HPV sequences.

Funding sources This study was initiated with funds from the BC Cancer Foundation and supported through a Research Sponsorship Agreement with Merck Frosst Canada Ltd. AB-W is a Michael Smith Foundation for Health Research Senior Scholar.

Competing interests This study was carried out by academic researchers and was funded by Merck Frosst Canada Ltd. A research agreement between Merck Frosst Canada Ltd. and the BC Cancer Agency gives control of all publication content to the academic researchers.

Potential conflict of interest Marc Brisson is a former employee of Merck Frosst Canada. No other potential conflicts of interest.

Open Access This article is distributed under the terms of the Creative Commons Attribution Noncommercial License which permits any noncommercial use, distribution, and reproduction in any medium, provided the original author(s) and source are credited.

\section{References}

1. Walboomers JM, Jacobs MV, Manos MM et al (1999) Human papillomavirus is a necessary cause of invasive cervical cancer worldwide. J Pathol 189:12-19. doi:10.1002/(SICI)1096-9896 (199909) 189:1<12::AID-PATH431>3.0.CO;2-F

2. Munoz N, Mendez F, Posso H et al (2004) Incidence, duration, and determinants of cervical human papillomavirus infection in a cohort of Colombian women with normal cytological results. J Infect Dis 190:2077-2087. doi:10.1086/425907

3. Harper DM, Franco EL, Wheeler C et al (2004) Efficacy of a bivalent $\mathrm{L} 1$ virus-like particle vaccine in prevention of infection with human papillomavirus types 16 and 18 in young women: a randomised controlled trial. Lancet 364:1757-1765. doi:10.1016/ S0140-6736(04)17398-4

4. Harper DM, Franco EL, Wheeler CM et al (2006) Sustained efficacy up to 4.5 years of a bivalent L1 virus-like particle vaccine against human papillomavirus types 16 and 18: follow-up from a randomised control trial. Lancet 367:1247-1255. doi: 10.1016/S0140-6736(06)68439-0

5. Koutsky LA, Ault KA, Wheeler CM et al (2002) A controlled trial of a human papillomavirus type 16 vaccine. $\mathrm{N}$ Engl J Med 347:1645-1651. doi:10.1056/NEJMoa020586

6. Villa LL, Costa RL, Petta CA et al (2005) Prophylactic quadrivalent human papillomavirus (types $6,11,16$, and 18) L1 viruslike particle vaccine in young women: a randomised double-blind placebo-controlled multicentre phase II efficacy trial. Lancet Oncol 6:271-278. doi:10.1016/S1470-2045(05)70101-7

7. Shi L, Sings HL, Bryan JT et al (2007) GARDASIL: prophylactic human papillomavirus vaccine development-from bench top to bed-side. Clin Pharmacol Ther 81:259-264. doi:10.1038/sj.clpt. 6100055

8. SurePath (2005) British Columbia Cervical Cancer Screening Program Feasibility Study: SurePath TM Liquid-Based Cytology: BC Cancer Agency

9. de Roda Husman AM, Walboomers JM, van den Brule AJ, Meijer CJ, Snijders PJ (1995) The use of general primers GP5 and GP6 elongated at their $3^{\prime}$ ends with adjacent highly conserved sequences improves human papillomavirus detection by PCR. J Gen Virol 76(Pt 4):1057-1062. doi:10.1099/0022-1317-76-41057

10. Asato T, Maehama T, Nagai Y, Kanazawa K, Uezato H, Kariya K (2004) A large case-control study of cervical cancer risk associated with human papillomavirus infection in Japan, by nucleotide sequencing-based genotyping. J Infect Dis 189:1829-1832. doi:10.1086/382896

11. Young TK, McNicol P, Beauvais J (1997) Factors associated with human papillomavirus infection detected by polymerase chain reaction among urban Canadian aboriginal and non-aboriginal women. Sex Transm Dis 24:293-298. doi:10.1097/00007435199705000-00011

12. CCSP (2004) British Columbia Cervical Cancer Screening Program 2004 annual report: BC Cancer Agency

13. Dunne EF, Unger ER, Sternberg M et al (2007) Prevalence of HPV infection among females in the United States. Jama 297:813-819. doi:10.1001/jama.297.8.813

14. Brown DR, Rawlings K, Handy V et al (1996) Human papillomavirus detection by hybrid capture in paired cervicovaginal lavage and cervical biopsy specimens. J Med Virol 48:210-214. doi:10.1002/(SICI)1096-9071(199602)48:2<210::AID-JMV15>3. $0 . \mathrm{CO} ; 2-\mathrm{I}$

15. Sellors JW, Lorincz AT, Mahony JB et al (2000) Comparison of self-collected vaginal, vulvar and urine samples with physiciancollected cervical samples for human papillomavirus testing to detect high-grade squamous intraepithelial lesions. Cmaj 163: 513-518

16. Ogilvie GS, Patrick DM, Schulzer M et al (2005) Diagnostic accuracy of self collected vaginal specimens for human papillomavirus compared to clinician collected human papillomavirus specimens: a meta-analysis. Sex Transm Infect 81:207-212. doi: 10.1136/sti.2004.011858

17. Pham TH, Nguyen TH, Herrero R et al (2003) Human papillomavirus infection among women in South and North Vietnam. Int J Cancer 104:213-220. doi:10.1002/ijc.10936

18. Castellsague X, Menendez C, Loscertales MP et al (2001) Human papillomavirus genotypes in rural Mozambique. Lancet 358: 1429-1430. doi:10.1016/S0140-6736(01)06523-0

19. Sellors JW, Mahony JB, Kaczorowski J et al (2000) Prevalence and predictors of human papillomavirus infection in women in 
Ontario, Canada. Survey of HPV in Ontario Women (SHOW) group. Cmaj 163:503-508

20. Coupé VM, Berkhof J, Bulkmans NW, Snijders PJ, Meijer CJ (2008) Age-dependent prevalence of 14 high-risk HPV types in The Netherlands: implications for prophylactic vaccination and screening. Br J Cancer 98(3):646-651. doi:10.1038/sj.bjc. 6604162

21. Bao YP, Li N, Smith JS, Qiao YL, ACCPAB members (2008) Human papillomavirus type distribution in women from Asia: a meta-analysis. Int J Gynecol Cancer 18(1):71-79

22. Sukvirach S, Smith JS, Tunsakul S et al (2003) Population-based human papillomavirus prevalence in Lampang and Songkla, Thailand. J Infect Dis 187:1246-1256. doi:10.1086/373901

23. Thomas JO, Herrero R, Omigbodun AA et al (2004) Prevalence of papillomavirus infection in women in Ibadan, Nigeria: a population-based study. Br J Cancer 90:638-645. doi:10.1038/ sj.bjc. 6601515

24. Molano M, van den Brule AJ, Posso H et al (2002) Low grade squamous intra-epithelial lesions and human papillomavirus infection in Colombian women. Br J Cancer 87:1417-1421. doi: 10.1038/sj.bjc. 6600650

25. de Sanjose S, Almirall R, Lloveras B et al (2003) Cervical human papillomavirus infection in the female population in Barcelona, Spain. Sex Transm Dis 30:788-793. doi:10.1097/01.OLQ. 0000080177.82204.E0

26. Matos E, Loria D, Amestoy GM et al (2003) Prevalence of human papillomavirus infection among women in Concordia, Argentina: a population-based study. Sex Transm Dis 30:593-599. doi: 10.1097/01.OLQ.0000085181.25063.6C

27. Shin HR, Lee DH, Herrero R et al (2003) Prevalence of human papillomavirus infection in women in Busan, South Korea. Int $\mathbf{J}$ Cancer 103:413-421. doi:10.1002/ijc.10825
28. Clifford GM, Gallus S, Herrero R et al (2005) Worldwide distribution of human papillomavirus types in cytologically normal women in the International Agency for Research on Cancer HPV prevalence surveys: a pooled analysis. Lancet 366:991-998. doi: 10.1016/S0140-6736(05)67069-9

29. Vernon SD, Unger ER, Williams D (2000) Comparison of human papillomavirus detection and typing by cycle sequencing, line blotting, and hybrid capture. J Clin Microbiol 38:651-655

30. Manhart LE, Holmes KK, Koutsky LA et al (2006) Human papillomavirus infection among sexually active young women in the United States: implications for developing a vaccination strategy. Sex Transm Dis 33:502-508. doi:10.1097/01.olq.0000 204545.89516.0a

31. Sellors JW, Karwalajtys TL, Kaczorowski JA et al (2002) Prevalence of infection with carcinogenic human papillomavirus among older women. Cmaj 167:871-873

32. Feoli-Fonseca JC, Oligny LL, Brochu P, Simard P, Falconi S, Yotov WV (2001) Human papillomavirus (HPV) study of 691 pathological specimens from Quebec by PCR-direct sequencing approach. J Med Virol 63:284-292. doi:10.1002/1096-9071 (200104)63:4<284::AID-JMV1003>3.0.CO;2-H

33. Richardson H, Kelsall G, Tellier P et al (2003) The natural history of type-specific human papillomavirus infections in female university students. Cancer Epidemiol Biomarkers Prev 12:485490

34. Chan PK, Cheung TH, Tam AO et al (2006) Biases in human papillomavirus genotype prevalence assessment associated with commonly used consensus primers. Int J Cancer 118:243-245. doi:10.1002/ijc.21299 